\title{
Variational Method Integrating Edge Detection and Smoothing in Digital Images
}

\author{
Italo M. F. Santos, Abimael D. Loula, \\ Gilson A. Giraldi \\ Dept. of Math. and Comp. Methods \\ National Laboratory for Scientific Computing \\ Petrópolis, Brazil \\ \{italof,aloc,gilson\}@lncc.br
}

\author{
Gastão F. Miranda Junior \\ Dept. de Matemática \\ Universidade Federal de Sergipe \\ Aracaju, Brazil \\ gastao@mat.ufs.br
}

\author{
Paulo S. S. Rodrigues \\ Dept. de Engenharia Elétrica \\ FEI University Center \\ São Bernardo do Campo, Brazil \\ psergio@fei.edu.br
}

\begin{abstract}
There is a consensus in computer vision about the importance of the scale concept for edge extraction and for image smoothing or representation. In this paper we explore a variational approach that allows to put together edge detection and image smoothing in a unified linear scheme. Basically, the functional proposed by Mumford and Shah is re-written as an energy defined with two arguments: the first one representing smooth versions of the original image and the second one encompassing its edge set. We follow known results in the variational analysis to obtain a numerical scheme to minimize the energy. We apply Fourier analysis to verify that the iterative scheme converges to a low-pass representation of the original image in the first argument and a high-pass signal in the other one. In the experimental results we show that the obtained scheme encourages intraregion image smoothing in preference to interregion blurring with edge localization at a desired scale.

Index Terms-smoothing, edge extraction, scale, variational methods
\end{abstract}

\section{INTRODUÇÃO}

Em visão computacional, as representações hierárquicas para imagens permitem simplificar processos, tais como, extração de bordas e segmentação. Neste contexto, a noção de escala tem um papel fundamental [1]. Matematicamente, este conceito levou aos métodos de representação multi-escala os quais, no caso linear, imergem a imagem em uma família de funções obtidas pela convolução da imagem original com o núcleo Gaussiano [2]. Esta metodologia pode ser formulada em termos da equação de difusão do calor, cuja forma anisotrópica e não-linear originou o método de Perona-Malik [3], ponto de partida para os aos métodos multi-escalas nãolineares baseados em equações diferenciais parciais [4], [5].

Um ponto fundamental nestes métodos é o efeito da suavização sobre os pontos de borda da imagem. A medida que vamos aumentado a escala (suavização) mais detalhe é perdido na imagem. O desejável é que este processo tenha algum mecanismo de controle sobre a 'mistura' entre objeto de interesse e fundo da imagem. Esta propriedade é a grande vantagem dos espaços de escala não-lineares sobre os lineares, pois, nestes últimos a suavização não é seletiva, dificultado a localização das bordas ao longo da representação hierárquica [3].

Thanks for CNPq and CAPES.
Esta discussão leva ao conceito recente de suavização com reconhecimento de bordas (edge-aware smoothing - EAS), que pode ser implementado seguindo a linha de métodos de difusão não-linear ou, iterativamente, adicionar as informações de borda de volta a uma imagem suavizada [6].

A proposta de combinar informações de borda com a imagem durante o processo de suavização remete a formulações variacionais baseadas no trabalho de Mumford e Shah [7]. O funcional proposto por Mumford e Shah permite colocar matematicamente as versões passa-baixa (imagem suavizada) e passa-alta (conjunto de pontos de borda) da imagem em espaços funcionais convenientes, abrindo caminho para um método de processamento de imagens do tipo EAS, que possui duas propriedades adicionais [8], [9]: (i) Convergência para imagem contínua por partes e mapa de arestas; (b) Principio de Causalidade: não são gerados artefatos quando aumentamos a escala. Apesar destas vantagens, a exploração dos métodos propostos em [8], [9] para processamento de imagens ficou restrita a poucos casos apresentados nestas referências, provavelmente devido ao custo computacional e a complexidade dos métodos variacionais envolvidos.

Neste trabalho, partimos da formulação variacional apresentada em [8] e discutimos extensivamente sua aplicação no contexto de EAS e detecção de bordas. Para isso, seguimos o esquema usado em [8], onde a imagem suavizada e seu conjunto de bordas são representados por funções $(u, v)$, definidas em um espaço funcional conveniente. Nesta formulação, a energia do modelo de Mumford e Shah é convertida em um funcional $E(u, v)$, usando artifícios de $\Gamma$-convergência. A minimização de $E(u, v)$ é obtida por um esquema numérico iterativo, totalmente linear, o que possibilita a utilização dos métodos de diferenças finitas ou elementos finitos, com propriedades numéricas de consistência e estabilidade. Nesta linha, as contribuições deste artigo são: (a) Desenvolvimento de um esquema de diferenças finitas que reduz custo computacional, se comparado com elementos finitos, e simplifica inicialização; (b) Análise no espaço de Fourier do método iterativo correspondente; (c) Análise da eficiência do método para imagens com textura e ruído; (d) Comparação com técnicas tradicionais: Perona-Malik [10] e Canny [11].

O texto está organizado como segue. Na seção II, apre- 
sentamos os fundamentos da técnica em foco. Em seguida, na seção III desenvolvemos o método proposto. Na seção IV apresentamos um estudo do método via análise de Fourier. Os experimentos computacionais são discutidos na seção V. As conclusões e trabalhos futuros são apresentados na seção VI.

\section{FUNDAMENTAÇÃO TEÓRICA}

Neste trabalho, uma imagem digital com domínio $\Omega \subset$ $\mathbb{R}^{2}$ é representada por uma função $g: \Omega \rightarrow[0,1] \subset$ $\mathbb{R}$, sendo $g$ uma função quadrado integrável $(g \in$ $L^{2}(\Omega)$ ). A premissa é determinar $u_{\min } \in H^{1}(\Omega)=$ $\left\{f \in L^{2}(\Omega) ; \frac{\partial f}{\partial x} \in L^{2}(\Omega)\right.$ e $\left.\frac{\partial f}{\partial y} \in L^{2}(\Omega)\right\}$, que minimiza o funcional $E(u)$ proposto por Mumford e Shah [7]. Para isto, em [8] utilizam-se recursos de $\Gamma$-convergência para definir um novo funcional $E_{c}: H^{1}(\Omega) \times H^{1}(\Omega) \rightarrow \mathbb{R}$, dado por:

$E_{c}(u, v)=\frac{1}{2} \int_{\Omega}\left\{\beta(u-g)^{2}+\left(v^{2}+k\right)\|\nabla u\|^{2}+M_{c}(v)\right\} d \Omega$

onde,

$$
M_{c}(v)=\frac{2 \alpha}{\pi}\left(c\|\nabla v\|^{2}+\frac{(1-v)^{2}}{4 c}\right),
$$

e $c$ é um número pequeno, $\alpha>0$ e $\beta>0$ são parâmetros, $\alpha \gg k$ controla o grau de suavização e $g \in H^{1}(\Omega)$ é a imagem original. Além disso, $E_{c} \rightarrow E$ quando $c \rightarrow 0$, de tal forma que as sequências minimizantes $u_{c}, v_{c}$ de $E_{c}$ convergem para $\left(u_{\text {min }}, v_{\text {min }}\right) \in H^{1}(\Omega) \times H^{1}(\Omega)$. Na próxima seção, discutiremos o significado de $u$ e $v$.

Partindo da formulação fraca para a otimização do funcional (1), utilizamos a diferencial de Gateaux [9] para obter as seguintes condições que devem ser satisfeitas por $(u, v) \in$ $H^{1}(\Omega) \times H^{1}(\Omega)$ :

$$
\begin{gathered}
\int_{\Omega}\left\{\|\nabla u\|^{2} v \hat{v}+\frac{\alpha}{\pi}\left(2 c \nabla v \cdot \nabla \hat{v}-\frac{(1-v) \hat{v}}{2 c}\right)\right\} d \Omega=0, \\
\int_{\Omega}\left\{\beta(u-g) \hat{u}+\left(v^{2}+k\right) \nabla u \cdot \nabla \hat{u}\right\} d \Omega=0,
\end{gathered}
$$

$\forall \hat{u}, \hat{v} \in H^{1}(\Omega)$ são denominadas funções de teste na formulação fraca.

A partir das expressões (2)-(3) podemos desenvolver um esquema iterativo para gerar uma sequência $\left(u_{n}, v_{n}\right)$ a qual tem a propriedade $u_{n} \rightarrow u_{\text {min }}$ e $v_{n} \rightarrow v_{\text {min }}$ quando $n \rightarrow \infty$. Neste caso, como apresentado em [8], é necessário fornecer condições iniciais $u_{0}$ e $v_{0}$ para o método.

\section{MÉTODO PROPOSTO}

Para efetuar a discretização do problema definido pelas expressões (2)-(3), precisamos extrair a forma forte de ambas as equações. Neste trabalho, diferentemente de [8], propomos um esquema numérico que necessita apenas da condição inicial $u_{0}$, definida pela imagem de entrada. Para isso, começaremos pela equação (2), que será utilizada para calcular $v_{n}$ a partir de $u_{n-1}$. Assim, utilizando o teorema de Stokes para obter a forma forte e substituindo $u$ e $v$ por $u_{n-1}$ e $v_{n}$, respectivamente, podemos converter a expressão (2) na forma:

$$
\begin{aligned}
& \int_{\Omega}\left\{\left\|\nabla u_{n-1}\right\|^{2} v_{n}-\frac{\alpha}{\pi}\left(\frac{\left(1-v_{n}\right)}{2 c}+2 c \cdot d i v\left(\nabla v_{n}\right)\right)\right\} \hat{v} d \Omega \\
& \quad+\int_{\partial \Omega} \frac{\partial v_{n}}{\partial \vec{n}} \hat{v} d \partial \Omega=0,
\end{aligned}
$$

$\forall \hat{v} \in H^{1}(\Omega)$, cuja solução $v_{n}$ pode ser obtida resolvendo o seguinte problema de valor de contorno:

$$
\left\{\begin{array}{l}
\left\|\nabla u_{n-1}\right\|^{2} v_{n}-\frac{\alpha}{\pi}\left(\frac{\left(1-v_{n}\right)}{2 c}+2 c \cdot d i v\left(\nabla v_{n}\right)\right)=0, \\
\text { sujeito a : } \\
\frac{\partial v_{n}}{\partial \vec{n}}=0, \text { na fronteira } \partial \Omega .
\end{array}\right.
$$

Procedendo analogamente para a expressão (3), obtemos:

$$
\begin{aligned}
& \int_{\Omega}\left\{\beta\left(u_{n}-g\right)-\operatorname{div}\left(\left(v_{n-1}^{2}+k\right) \nabla u_{n}\right)\right\} \hat{u} d \Omega \\
+ & \int_{\partial \Omega}\left(v_{n-1}^{2}+k\right) \frac{\partial u_{n}}{\partial \vec{n}} \hat{u} d \partial \Omega=0,
\end{aligned}
$$

$\forall \hat{u} \in H^{1}(\Omega)$, e, consequentemente, podemos obter $u_{n}$ pela solução do seguinte problema:

$$
\left\{\begin{array}{l}
\beta\left(u_{n}-g\right)-\operatorname{div}\left(\left(v_{n}^{2}+k\right) \nabla u_{n}\right)=0 \\
\text { sujeito a : } \\
\frac{\partial u_{n}}{\partial \vec{n}}=0, \text { na fronteira } \partial \Omega
\end{array}\right.
$$

No esquema iterativo definido pelas expressões (5)-(7), basta a condição inicial $u_{0}$, pois, a solução $v_{1}$ obtida pela expressão (5) será utilizada para calcular $u_{1}$ através da solução do problema (7), e assim por diante para $n>1$.

Para resolver numericamente as equações (5)-(7) utilizamos diferenças finitas computadas na malha definida pelas posições $(i, j)$ dos pixeis da imagem. Com base no artigo [12], escolhemos as seguintes discretizações para obter um esquema numérico com a precisão necessária:

$$
\begin{aligned}
\left(\left(\partial_{x}^{+} u_{n-1}^{i, j}\right)^{2}+\left(\partial_{y}^{+} u_{n-1}^{i, j}\right)^{2}\right) v_{n}^{i, j} & \\
-\frac{\alpha}{\pi}\left(\frac{\left(1-v_{n}^{i, j}\right)}{2 c}+2 c\left(\partial_{x}^{-} \partial_{x}^{+} v_{n}^{i, j}+\partial_{y}^{-} \partial_{y}^{+} v_{n}^{i, j}\right)\right) & =0 \\
\beta\left(u_{n}^{i, j}-g^{i, j}\right)-\left(\partial_{x}^{-}\left(\left(\left(v_{n}^{i, j}\right)^{2}+k\right) \partial_{x}^{+}\left(u_{n}^{i, j}\right)\right)\right. & \\
\left.+\partial_{y}^{-}\left(\left(\left(v_{n}^{i, j}\right)^{2}+k\right) \partial_{y}^{+}\left(u_{n}^{i, j}\right)\right)\right) & =0
\end{aligned}
$$

onde $\partial_{x}^{-}$e $\partial_{x}^{+}$denotam operadores usuais de diferencias finitas adiantada e atrasada, respectivamente (idem para $\partial_{y}^{-}$e $\partial_{y}^{+}$), sendo $u_{n}^{i, j} \equiv u_{n}(i, j)$. Para forçar a condição de contorno para $u_{n}$ em $\partial \Omega$ fazemos: $u_{n}^{-1, j}=u_{n}^{0, j} ; u_{n}^{N+1, j}=u_{n}^{N, j} ; u_{n}^{i,-1}=$ $u_{n}^{i, 0} ; u_{n}^{i, M+1}=u_{n}^{i, M}$, onde $N$ e $M$ são a quantidade de pixeis da imagem de entrada $g$ nas direções $x$ e $y$ respectivamente (procedimento análogo é realizado para $u_{n}$ e $v_{n}$ ). Desta forma, o sistema de equações acima pode ser escrito na forma linear $A_{1} v_{n}=F_{1}$ e $A_{2} u_{n}=F_{2}(g)$, onde $A_{1}=A_{1}\left(u_{n-1}\right), F_{1}$ é um vetor constante e $A_{2}=A_{2}\left(v_{n}\right)$, sendo portanto, um sistema 
de equações lineares com $2(N \cdot M)$ incógnitas. O critério de parada é computado pela norma do máximo via expressão:

$$
\max _{0 \leq i \leq N ; 0 \leq j \leq M}\left|v_{i, j}^{n}-v_{i, j}^{n-1}\right|<\varepsilon,
$$

onde $\varepsilon$ é uma tolerância previamente definida.

\section{ANÁLISE DE FOURIER}

Para verificar que $u_{n}$ e $v_{n}$ no esquema (5)-(7) representam a imagem suavizada e conjunto de pontos de borda, respectivamente, vamos desconsiderar efeitos da fronteira $\partial \Omega$ e tomar a transformada de Fourier, denotada por $\mathbb{F}$, da expressão (5), usando o fato de que, se $\mathbb{F}(f(x, y))=\widehat{f}\left(\omega_{1}, \omega_{2}\right)$ então $\mathbb{F}(\partial f / \partial x)=j \omega_{1} \hat{f}\left(\omega_{1}, \omega_{2}\right)$ e $\mathbb{F}(\partial f / \partial y)=j \omega_{2} \hat{f}\left(\omega_{1}, \omega_{2}\right)$, onde $j^{2}=-1$. Desta forma, a expressão (5) pode ser escrita, no domínio de frequência, como:

$\mathbb{F}\left(\left\|\nabla u_{n-1}\right\|^{2} v_{n}\right)-\frac{2 c \alpha}{\pi}\left(\omega_{1}^{2}+\omega_{2}^{2}\right) \mathbb{F}\left(v_{n}\right)=\frac{\alpha}{\pi 2 c} \mathbb{F}\left(1-v_{n}\right)$.

Usando agora a propriedade $\mathbb{F}\left(\left\|\nabla u_{n-1}\right\|^{2} v_{n}\right)=$ $\mathbb{F}\left(\left\|\nabla u_{n-1}\right\|^{2}\right) \otimes \mathbb{F}\left(v_{n}\right)$, onde ' $\otimes$ ' denota convolução, e considerando apenas uma vizinhança da origem no domínio de frequências, a expressão acima toma a forma:

$$
\mathbb{F}\left(\left\|\nabla u_{n-1}\right\|^{2}\right) \otimes \mathbb{F}\left(v_{n}\right) \approx \frac{\alpha}{\pi 2 c} \mathbb{F}\left(1-v_{n}\right) .
$$

Sabendo que $\left\|\nabla u_{n-1}\right\|^{2}$ é uma filtragem passa-alta de $u_{n-1}$, podemos supor $\mathbb{F}\left(\left\|\nabla u_{n-1}\right\|^{2}\right) \approx 0$ próximo da origem, consequentemente, $\mathbb{F}\left(1-v_{n}\right) \approx 0$ nesta região indicando filtragem passa-alta de $u_{n-1}$ para a versão negativa da imagem $v_{n}$. Procedendo analogamente para a equação (7), usando as propriedades acima citadas e o fato da transformada de Fourier ser um operador linear, obteremos:

$$
\begin{gathered}
\beta \mathbb{F}\left(u_{n}\right)-\beta \mathbb{F}(g)-j \omega_{1} \mathbb{F}\left(v_{n}^{2} \frac{\partial u_{n}}{\partial x}\right)-j \omega_{2} \mathbb{F}\left(v_{n}^{2} \frac{\partial u_{n}}{\partial y}\right) \\
+k\left(\omega_{1}^{2}+\omega_{2}^{2}\right) \mathbb{F}\left(u_{n}\right)=0,
\end{gathered}
$$

Para simplificar a análise desta expressão, vamos admitir $\mathbb{F}\left(v_{n}^{2} \frac{\partial u_{n}}{\partial x}\right) \approx \mathbb{F}\left(v_{n}^{2}\right) \mathbb{F}\left(\frac{\partial u_{n}}{\partial x}\right)=j \omega_{1} \mathbb{F}\left(u_{n}\right) \mathbb{F}\left(v_{n}^{2}\right) \mathrm{e}$ $\mathbb{F}\left(v_{n}^{2} \frac{\partial u_{n}}{\partial y}\right) \approx \mathbb{F}\left(v_{n}^{2}\right) \mathbb{F}\left(\frac{\partial u_{n}}{\partial y}\right)=j \omega_{2} \mathbb{F}\left(u_{n}\right) \mathbb{F}\left(v_{n}^{2}\right)$. Então, inserindo estas aproximações na expressão (12) e isolando $\mathbb{F}\left(u_{n}\right)$ obtemos:

$$
\mathbb{F}\left(u_{n}\right)=\frac{\beta \mathbb{F}(g)}{\beta+k\left(\omega_{1}^{2}+\omega_{2}^{2}\right)+\mathbb{F}\left(v_{n}^{2}\right)\left(\omega_{1}^{2}+\omega_{2}^{2}\right)},
$$

o que indica uma filtragem tipo passa-baixa da imagem original $g$, modulada pelo parâmetro $\beta$ e a transformada de Fourier da função $v_{n}^{2}$.

\section{RESULTADOS COMPUTACIONAIS}

Nesta seção apresentamos experimentos computacionais para discutir aspectos qualitativos, sensibilidade do modelo em relação a ruído e parâmetros, comparações entre o método proposto e dois métodos tradicionais: Perona-Malik [10] e Canny [11]. Seguindo a metodologia da seção III, a inicialização do esquema iterativo definido pelas expressões (8)-(9) é feita usando a imagem de entrada. Cada iteração do método demanda obter uma solução $v_{n}$ da equação (8) e uma solução $u_{n}$ da expressão (9). Os parâmetros $c, \alpha, \beta, k$ e $\varepsilon$ são determinados por experimentos numéricos.

Inicialmente, vamos verificar a capacidade do método para suavizar as regiões de interesse preservando as bordas dos objetos na presença de ruído. Para isso, consideramos a imagem da Figura 1.(i) e adicionamos ruído Gaussiano com variância $\sigma=0.1$ e média $\mu=0.0$, gerando a imagem mostrada na Figura 1.(ii). Os resultados do esquema iterativo (8)-(9), tendo como entrada a imagem 1.(ii), podem ser visualizados nas Figuras 1.(iii)-(iv).

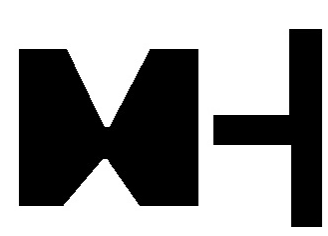

(i)

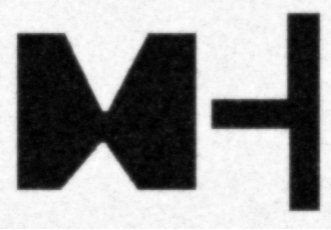

(iii)

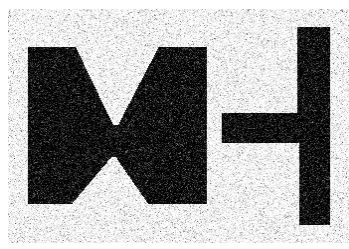

(ii)

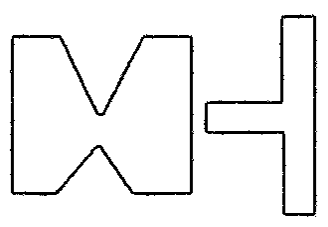

(iv)
Fig. 1. (i) Imagem original. (ii) Imagem corrompida com ruído Gaussiano $(\mu, \sigma)=(0.0,0.1)$. (iii) Imagem suavizada $u$ obtida após cinco iterações. (iv) Mapa de bordas $v$ gerado. Parâmetros: $c=0.001, \alpha=0.01, \beta=0.7$, $k=1.0$, e tolerância $\varepsilon=10^{-2}$ na equação (10).

A análise visual mostra boa qualidade da imagem restaurada (Figura 1.(iii)) com pouco borramento na região das bordas. É importante ressaltar também a diminuição do ruído, o que fica mais evidente na região mais clara da Figura 1.(iii). Com relação ao mapa de bordas mostrado na imagem da Figura 1.(iv), devemos observar também pouca interferência do ruído, tanto na região da borda dos objetos quanto em regiões distantes destas.

No próximo teste, utilizamos uma imagem (Figura 2.(i)) onde observamos regiões bem definidas (pupila, por exemplo) bem como detalhes sutis, tais como as estruturas internas na íris. O resultado do método iterativo aplicado sobre a Figura 2.(i) é mostrado nas imagens das Figuras 2.(ii) e 2.(iii). Com relação à versão suavizada da imagem original, podese observar a preservação dos detalhes acima mencionados, ressaltando mais uma vez a capacidade do método de priorizar suavização intra-objeto minimizando a 'mistura' entre um objeto e sua vizinhança. O mapa de bordas mostrado na Figura 2.(iii), consegue definir com precisão a fronteira da pupila e parcialmente a fronteira externa da esclerótica, localizada próxima da pálpebra. No caso das demais regiões, as bordas não ficaram bem definidas em função da presença de bordas subjetivas, o que pode ser visualmente observado na Figura 
2.(iv). Este exemplo será discutido novamente ao longo desta seção.

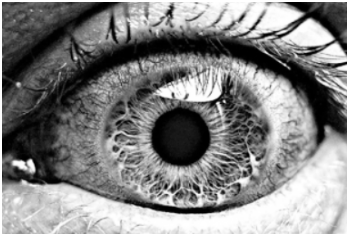

(i)

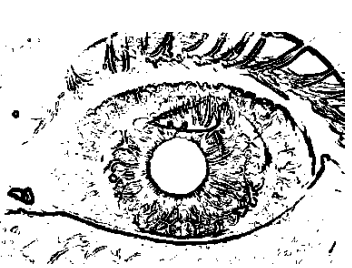

(iii)

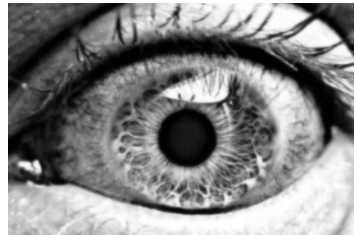

(ii)

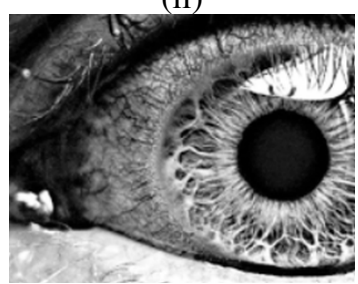

(iv)
Fig. 2. (i) Imagem original. (ii) Imagem suavizada após três iterações do esquema (8)-(9). (iii) Mapa de bordas. (iv) Realce da região esquerda do olho mostrando bordas subjetivas. Parâmetros: $c=0.001, \alpha=0.01, \beta=1.0$, $k=0.001$, tolerância $\varepsilon=10^{-2}$.

Para mostrar resultados mais consistentes com respeito a suavização efetuamos uma análise quantitativa através da medida SSIM (measure of structural similarity) [13]. Os valores da SSIM para a técnica proposta e para o método de Perona-Malik são apresentados na Tabela I, indicando superioridade do método apresentado nos dois primeiros casos. Por exemplo, os valores na primeira linha da tabela foram gerados computando o SSIM entre a imagem 3.(i) e as imagens suavizadas 3.(iii) e 3.(iv). Idem para as demais linhas da Tabela I. Os resultados de todos os experimentos usados para gerar a Tabela I podem ser visualizados na Figura 3. Os resultados do método abordado, mostrados nas imagens 3.(iii),(vii),(xi) foram obtidos com parâmetros: $c=0.001, \alpha=0.01, \beta=0.7$, $k=1.0$, com três iterações do esquema (8)-(9), para tolerância $\varepsilon=10^{-2}$ na condição de parada (10). Para o método de Perona-Malik [3], [10], os melhores resultados obtidos podem ser visualizados nas Figuras 3.(iv),(viii),(xii), com parâmetros $K=1, \Delta t=0.14$, com 5 interações, ajustados por tentativa e erro procurando maximizar o SSIM.

TABLE I

TABELA DE VALORES PARA A MÉTRICA SSIM.

\begin{tabular}{|c|c|c|}
\hline Figura & Perona-Malik & Método Proposto \\
\hline Fig. 3.(i) & 0.55 & 0.56 \\
\hline Fig. 3.(v) & 0.48 & 0.50 \\
\hline Fig. 3.(ix) & 0.59 & 0.57 \\
\hline
\end{tabular}

Pela análise visual das imagens na Figura 3, notamos que ambos os métodos possuem desempenho semelhante em relação à suavização. Porém, comparando as Figuras 3.(iii),(iv), observamos que o método proposto obteve mais suavização sem perda de detalhes importantes (aba do chapéu, por exemplo). Por outro lado, nas Figuras 3.(vii),(viii) vemos

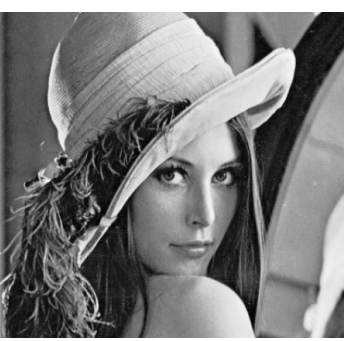

(i)

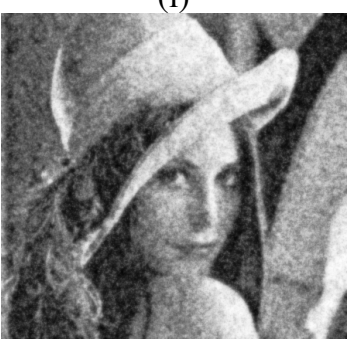

(iii)

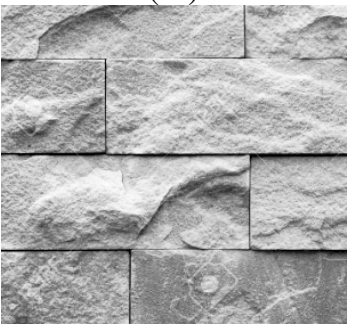

(v)

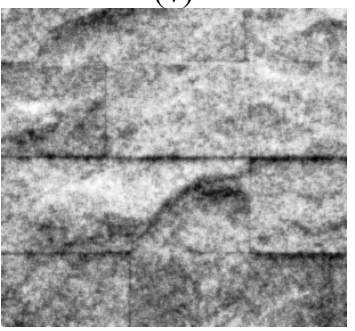

(vii)

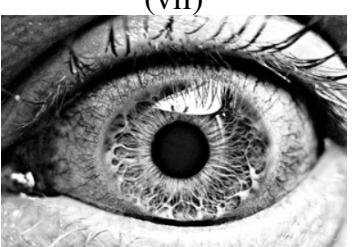

(ix)

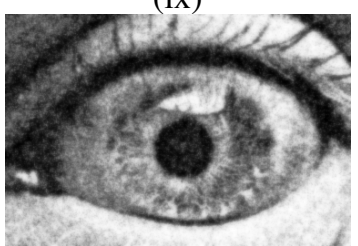

(xi)

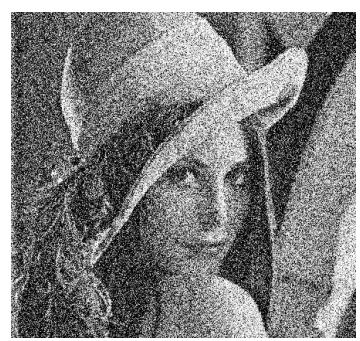

(ii)

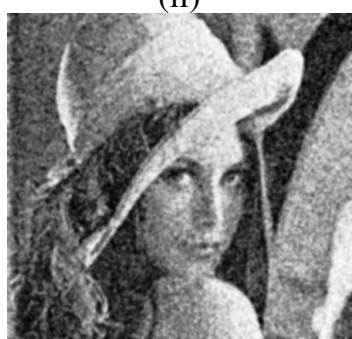

(iv)

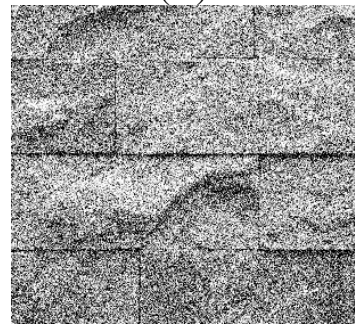

(vi)

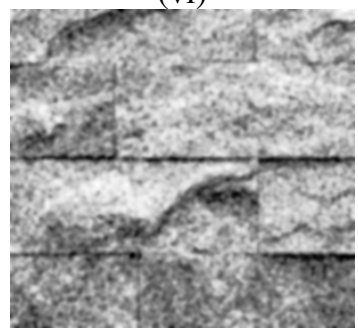

(viii)

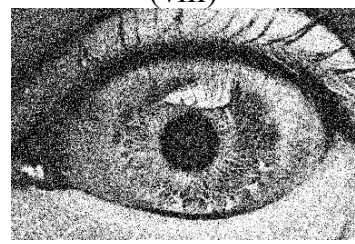

(X)

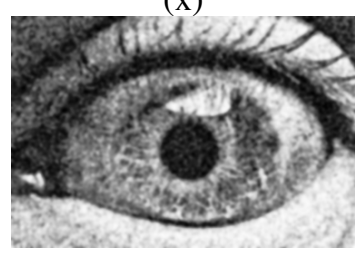

(xii)
Fig. 3. (i),(v),(ix) Imagens originais. (ii),(vi),(x) Imagens corrompidas por ruído Gaussiano $(\mu, \sigma)=(0.0,0.1)$. (iii),(vii),(xi) Imagem suavizada pelo método proposto. (iv),(viii),(xii) Imagem suavizada por Perona-Malik. 
que o método apresentado preservou melhor os frisos entre os blocos. Outro fator a ser considerado é que o método proposto converge para imagem contínua por partes e mapa de bordas [8], o que torna a escolha do critério de parada bem menos determinante no resultado, se comparado com Perona-Malik. Além disso, comparando os resultados das Figuras 2.(ii) e 3.(xi), nota-se que às duas imagens preservam as estruturas principais do olho, o que indica robustez do método em relação ao ruído.

É importante ressaltar que todos os resultados da Figura 3 , obtidos pelo nosso método, foram gerados com o mesmo conjunto de parâmetros explicitado acima, o que mostra a generalidade da técnica definida pelas expressões (8)-(9), em relação à escolha de valores para os parâmetros, considerando as diferentes características das imagens de entrada. Vale a pena observar que a expressão (13) indica influência importante do parâmetro $\beta$, uma vez que este aparece como fator no numerador, multiplicando a transformada da imagem de entrada $g$, bem como um termo extra no denominador. Para verificar essa influência, na Figura 4.(i) apresentamos um gráfico referente a influência do parâmetro $\beta$ na métrica SSIM, para as versões suavizadas da imagem 3.(x) obtidas ao variar apenas este parâmetro com os demais fixos em $c=0.001$, $\alpha=0.01, k=1.0$, tolerância $\varepsilon=10^{-2}$. Pelo gráfico da Figura 4.(i) observamos um ponto de máximo em torno de $\beta=0.7$ enquanto o pior caso ocorre para $\beta=0.1$. O número de iterações foi 3 nos dois casos, sendo evidente que a imagem 4.(ii) preserva mais detalhes da imagem original (Figura 3.(ix)) em comparação com Figura 4.(iii), embora o nível de ruído na primeira seja visualmente mais perceptível do que na segunda.

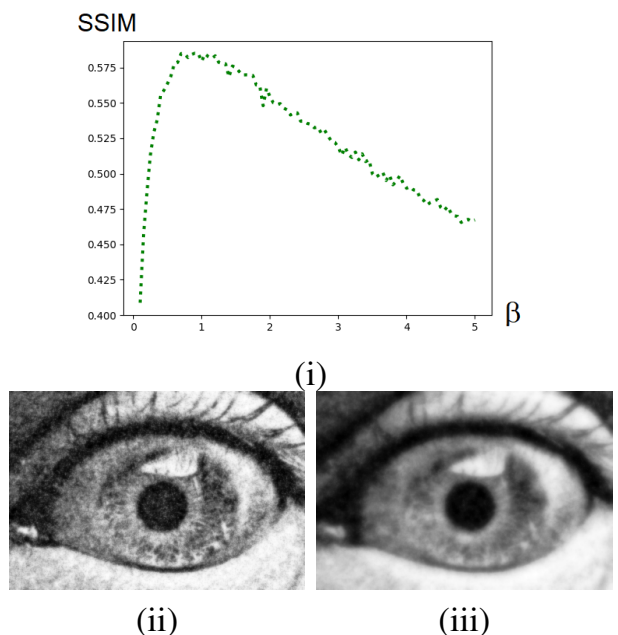

Fig. 4. (i) Relação entre $\beta$ e métrica SSIM. (ii) Melhor caso (SSIM máximo): $\beta=0.7$. (iii) Imagem suavizada para $\beta=0.1$ : valor mínimo de SSIM.

Nesta etapa, faremos a comparação entre os mapas de bordas provenientes do método apresentado, filtro gradiente aplicado na imagem suavizada por Perona-Malik (PeronaMalik+Gradiente) e Filtro de Canny. Dividimos os testes em imagens com textura e predominância de regiões nãohomogêneas (Figura 5.(i)-(ii)) e imagens que incluem tanto regiões complexas quanto com padrões de intensidade quase uniforme (Figura 6.(i)-(ii)). Para o método em questão foi utilizado os parâmetros: $c=0.001, \alpha=0.01, \beta=1.0$, $k=0.001$. Para tolerância $\varepsilon=10^{-2}$, foram executadas três iterações do esquema (8)-(9). Foram considerados como pontos de borda todos os pixeis $(i, j)$ satisfazendo $v(i, j)>0.998$. No caso do Perona-Malik+Gradiente, inicialmente executamos o primeiro com os parâmetros: $K=1, \Delta t=0.14$, e número de iterações igual a 5 [10]. Em seguida, aplica-se o operador gradiente. Para o filtro de Canny são considerados pontos de borda aqueles com intensidade de gradiente no intervalo $[0.392,0.784]$. De maneira geral, a observação das imagens das Figuras 5 e 6 mostra que o método proposto preservou mais detalhes das bordas dos objetos que o método de PeronaMalik. Com relação à comparação com o filtro de Canny as imagens 5.(vii)-(viii) mostram uma quantidade excessiva de pontos de borda o que ocorre em menor intensidade no método apresentado. No caso da Figura 6.(ii), observamos que o resultado do esquema proposto (Figuras 6.(iv)) e do filtro de Canny (Figuras 6.(viii)) apresentam mapas de borda visualmente semelhantes.

Com relação ao parâmetro $k$, a expressão (13) permite supor que valores mais elevados implicam em maior suavização, com provável influência na localização das bordas. No caso da Figura 1, apesar do ruído, os objetos possuem poucos detalhes. Assim, o valor $k=1.0$ permitiu bom resultado para a suavização (Figura 1.(iii)) sem interferir significativamente no mapa de bordas (Figura 1.(iv)). Porém, no caso das imagens na Figura 5, observamos um nível de detalhe bem mais elevado das estruturas internas. Assim, tivemos que diminuir este parâmetro para $k=0.001$ de modo a obter os detalhes (pontos de borda) na escala desejada.

\section{Conclusão E Trabalhos Futuros}

Neste artigo revisamos o trabalho [8] para propor um esquema iterativo, com fundamentos em métodos variacionais, que integra suavização e detecção de pontos bordas. Os experimentos computacionais indicam robustez em relação a ruído bem como eficiência mais elevada, ou competitiva, em comparação com os métodos de Perona-Malik e Canny. A sensibilidade em relação à escolha de parâmetros foi analisada correlacionando resultados experimentais e teóricos, obtidos via análise de Fourier. A complexidade computacional de cada iteração do método está limitada superiormente pelo custo de resolver dois sistemas lineares, cada um da ordem do número de pixeis da imagem $(N \cdot M)$. Não temos uma implementação otimizada para analisar com segurança o tempo de CPU.

Como trabalhos futuros, faremos implementação em GPU do método proposto, sua integração com esquemas multiescala baseados em pirâmides e comparação com métodos mais recentes [14], utilizando outras bases de imagens, com exploração sistemática do espaço de parâmetros de cada método.

\section{REFERENCES}

[1] T. Lindeberg, Scale-space theory in computer vision. Kluwer academic publishers, 1994. 


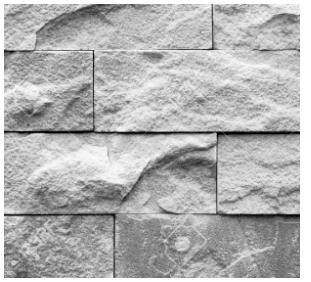

(i)

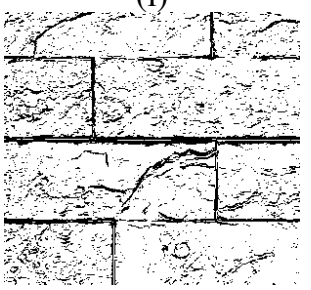

(iii)

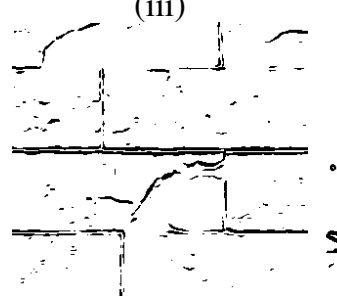

(v)

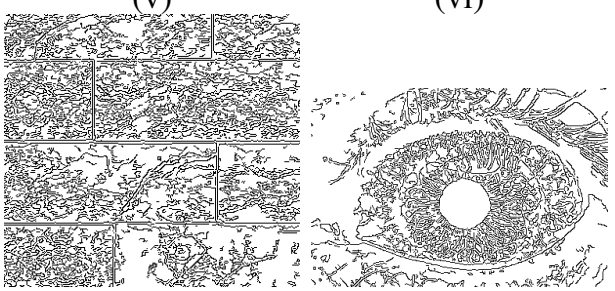

(vii)

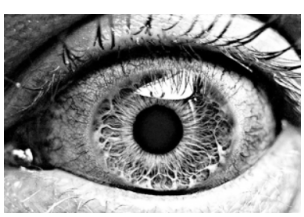

(ii)

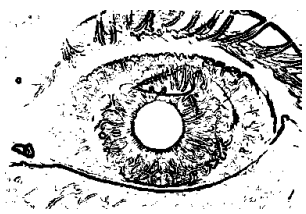

(iv)

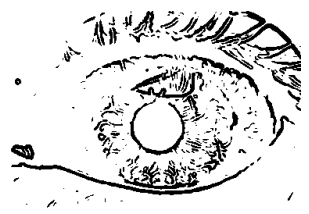

(vi)

(viii)
Fig. 5. (i)-(ii) Imagens originais. (iii)-(iv) Mapas de bordas do método trabalhado. (v)-(vi) Mapas de bordas do gradiente com Perona-Malik. (vii)(viii) Mapas de bordas do filtro de Canny.

[2] C. Lopez-Molina, B. D. Baets, H. Bustince, J. Sanz, and E. Barrenechea, "Multiscale edge detection based on gaussian smoothing and edge tracking," Knowledge-Based Systems, vol. 44, pp. 101 - 111, 2013.

[3] P. Perona and J. Malik, "Scale-space and edge detection using anisotropic diffusion," IEEE Trans. on patter analusis and Mach. Intell., vol. 12, no. 7, pp. 629-639, July 1990.

[4] L. Florack, A. Salden, B. T. H. Romery, J. Koenderink, and M. Viergever, "Nonlinear scale-space," Image and vision computing, vol. 13, no. 4, pp. 279-294, May 1995.

[5] C. Lopez-Molina and N. Madrid, "Non-linear scale-space based on fuzzy sharpening," in 2017 IFSA-SCIS, June 2017, pp. 1-6.

[6] M. Al-nasrawi, G. Deng, and B. S. Thai, "Edge-aware smoothing through adaptive interpolation," Signal, image and video processing, pp. $1-8,082017$.

[7] D. Mumford and J. Shah, "Optimal approximations by piecewise smooth functions and associated variational problems," Comm. on pure and applied mathematics, vol. 42, no. 5, pp. 577-685, 1989.

[8] B. Bourdin, "Image segmentation with a finite element method," ESAIM: Mathematical modelling and numerical analysis, vol. 33, no. 2, pp. 229244, 1999.

[9] T. J. Richardson and S. K. Mitter, "A variational formulation-based edge focussing algorithm," Sadhana, vol. 22, no. 4, pp. 553-574, Aug 1997.

[10] F. H. Fubel, Implementation Perona-Malik. github.com/fubel/PeronaMalikDiffusion, 2019.

[11] L. OpenCV, Canny filter at openCV. docs.opencv.org/3.1.0/da/d22/tutorial_py_canny.html, 2019.

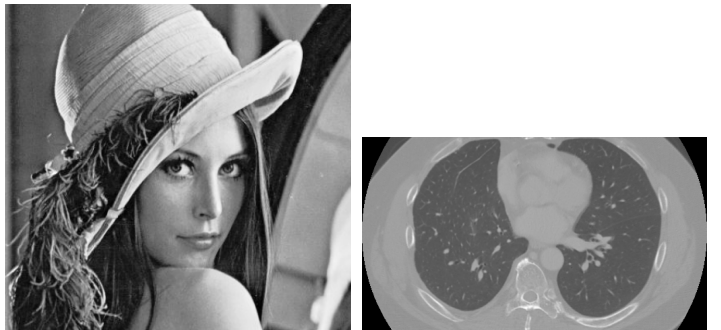

(ii)

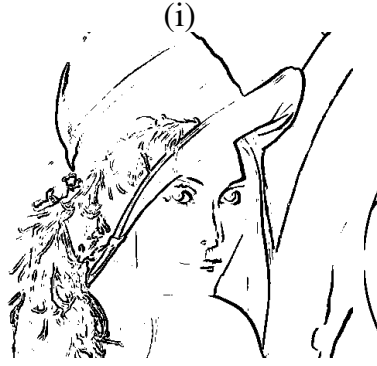

(iii)

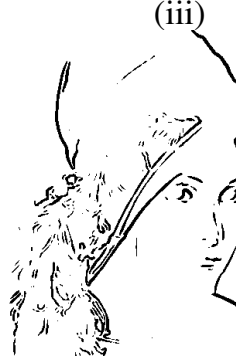

(v)

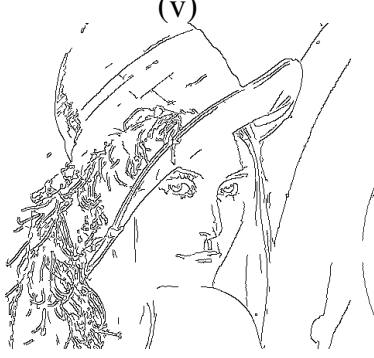

(vii)

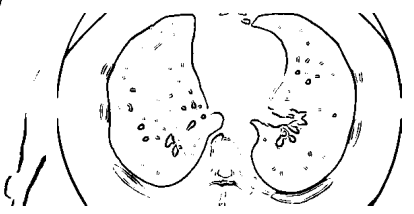

(iv)
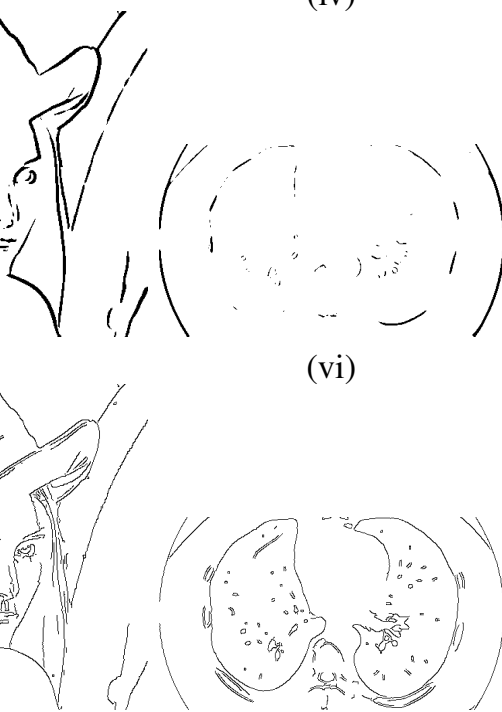

(viii)
Fig. 6. (i)-(ii) Imagens originais, contendo regiões quase homogêneas e com detalhes. (iii)-(vi) Mapas de bordas do método apresentado. (v)-(vi) Mapa de bordas do gradiente com Perona-Malik. (vii)-(viii) Mapa de bordas do filtro de Canny.

[12] I. M. F. Santos, G. F. M. Junior, and L. C. M. Aquino, "Segmentação de imagens pulmonares em tomografia computatorizada a partir do modelo de chan-vese com influência de ruído," in Anais do V ermac 2018, Maceió, 2018

[13] Z. Wang, A. C. Bovik, H. R. Sheikh, and E. P. Simoncelli, "Image quality assessment: from error visibility to structural similarity," IEEE Trans. on image processing, vol. 13, no. 4, pp. 600-612, 2004.

[14] G. Deng, "Guided wavelet shrinkage for edge-aware smoothing," IEEE Trans. on image processing, vol. 26, no. 2, pp. 900-914, Feb 2017. 